OPEN ACCESS

Edited by:

Leonard Wee,

Maastro Clinic, Netherlands

Reviewed by:

Laura Pala,

European Institute of Oncology (IEO),

lan Marschner,

The University of Sydney, Australia

*Correspondence:

Qitao Yu

447375272@qq.com

${ }^{\dagger}$ These authors have contributed equally to this work

Specialty section: This article was submitted to

Thoracic Oncology,

a section of the journal

Frontiers in Oncology

Received: 30 April 2021 Accepted: 02 August 2021 Published: 23 August 2021

Citation:

Zhao W, Jiang W, Wang $\mathrm{H}, \mathrm{He} J$, Su C and Yu Q (2021) Impact of Smoking History on Response to Immunotherapy in Non-Small-Cell

Lung Cancer: A Systematic Review and Meta-Analysis.

Front. Oncol. 11:703143.

doi: 10.3389/fonc.2021.703143

\section{Impact of Smoking History on Response to Immunotherapy in Non-Small-Cell Lung Cancer: A Systematic Review and Meta-Analysis}

\author{
Wenhua Zhao ${ }^{\dagger}$, Wei Jiang ${ }^{\dagger}$, Huilin Wang, Jianbo He, Cuiyun Su and Qitao Yu* \\ Department of Respiratory Oncology, Affiliated Tumor Hospital of Guangxi Medical University, Nanning, China
}

Objectives: To evaluate the impact of smoking history on the clinical benefit of immunotherapy in patients with non-small cell lung cancer (NSCLC).

Methods: Twenty-three randomized clinical trials and seven real-world studies were included in this meta-analysis. Hazard ratios (HRs) and 95\% confidence intervals (Cls) for overall survival (OS) and progression-free survival (PFS) and odds ratios for the overall response rate (ORR) were extracted. A fixed-effects or random-effects model was applied to obtain pooled estimates.

Results: Data from 16 high-quality trials involving 10,643 NSCLC patients receiving either immunotherapy or chemotherapy/placebo enabled direct comparison of the survival impact of smoking. Anti-PD-1/PD-L1/CTLA-4 immunotherapy was found to significantly prolong OS and PFS as compared to chemotherapy/placebo in smokers (HR for OS, 0.76 [0.69-0.83], $\mathrm{P}<0.00001$; HR for PFS, 0.65 [0.56-0.75], $\mathrm{P}<0.00001)$, and these trends were less or not significant in non-smokers (HR for OS, 0.91 [0.78-1.06], $P=0.25$; HR for PFS, 0.68 [0.45-1.03], P=0.07). Consistent results were obtained for the first-line or second/third-line use of immunotherapy and for non-squamous NSCLC patients only. Furthermore, the data from 7 trials and 7 real-world studies involving 4,777 patients receiving immunotherapy allowed direct comparison of therapeutic outcomes between smokers and non-smokers. Prolonged OS (HR 0.86 [0.75-0.99], P=0.04) and PFS (HR 0.69 [0.60-0.81], $P<0.0001$ ) and a higher response rate (ORR 1.20 [0.94-1.53], $P=0.15$ ) were observed in smokers compared to non-smokers receiving immunotherapy.

Conclusions: Immunotherapy was found to have a greater benefit in NSCLC patients with a smoking history than in those who had never smoked.

Keywords: immunotherapy, non-small cell lung cancer, smoking, clinical benefit, meta-analysis 


\section{INTRODUCTION}

Lung cancer remains a highly prevalent cancer and the leading cause of cancer-related death worldwide (1). Even with various therapeutic regimens available for NSCLC, the prognosis of many patients is still dismal. Continuous efforts have been made to improve outcomes in NSCLC patients, and some breakthroughs have been achieved in recent years, the most promising one being the development immunotherapy (2).

The basic approach of immunotherapy is to evoke an antitumor immune response by blocking an immune checkpoint, like programmed death-1/programmed death ligand-1 (PD-1/ PD-L1) and cytotoxic T-cell lymphocyte antigen-4, and thereby achieving durable control of a tumor (3). Although remarkable improvements in clinical outcomes in multiple cancer types have been achieved by immunotherapy (4), only a limited percentage of NSCLC patients respond to the therapy, with less than $30 \%$ of NSCLC patients benefiting from immunotherapy (5). Thus, efficient markers to identify NSCLC patients who are most likely to respond to immunotherapy are urgently needed.

Several biomarkers, such as PD-1/PD-L1 expression on tumor cells, T-cell infiltration within the tumor microenvironment, and tumor mutation burden (TMB) (6-8), have been proposed to be associated with the therapeutic response of NSCLC to immunotherapy. However, the acquisition of these parameters requires tumor biopsy, which is not always possible, especially for patients with advanced disease. Thus, the ability to identify responders based on clinical characteristics would be of great clinical significance. Smoking history is a risk factor for pulmonary carcinogenesis, and smoking is known to alter the immune microenvironment and TMB in lung cancer, effects which have been linked with the therapeutic efficacy of immunotherapy (7-10). However, direct evidence for whether a smoking history is associated with the response to immunotherapy is lacking. Previous studies have hinted that NSCLC patients who are smokers might derive greater benefit from immunotherapy as compared to those who are nonsmokers $(11,12)$. However, other reports have suggested that smoking history has no effect on the therapeutic outcome of immunotherapy $(13,14)$. The significance of smoking history in predicting the response to immunotherapy has yet to be systematically verified in a large-scale dataset.

Many clinical trials and real-world studies have provided data regarding immunotherapy-related outcomes and clinical characteristics like smoking history in NSCLC patients. However, these individual trials or studies have limited statistical power to validate a response difference related to smoking history. We thus carried out a meta-analysis by pooling the publicly available data to investigate the impact of smoking history on the therapeutic outcome of immunotherapy. We also evaluated the significance of smoking in different

Abbreviations: NSCLC, Non-small-cell Lung Cancer; ICI, Immune checkpoint inhibitor; PD-1, Programmed death-1; PD-L1, Programmed death ligand-1; CTLA-4, Cytotoxic T-lymphocyte-associated protein 4; OS, Overall survival; PFS, Progression-free survival; ICs, Confidence intervals; ORR, Overall response rate; HR, Hazard ratios; TMB, Tumor mutation burden. contexts (immunotherapy as first-line or not, adenocarcinoma or squamous cell carcinoma, etc.).

\section{MATERIALS AND METHODS}

\section{Literature Search Strategy and Study Selection}

We carried out a systematic search of the PubMed, Embase, and Cochrane databases as well as conference websites including the World Conference on Lung Cancer (WCLC), American Society of Clinical Oncology (ASCO), and European Society for Medical Oncology (ESMO) websites for articles or abstracts with no language restriction or limitation on publication year (up to September 10, 2020). Relevant studies were searched using the following terms: "(lung neoplasms OR non-small cell lung cancer) AND (pembrolizumab OR Keytruda OR MK-3475 OR SCH 900475 OR nivolumab OR Opdivo OR BMS-936558 OR MDX-1106 OR ONO-4538 OR atezolizumab OR Tecentriq OR MPDL3280A OR RG7446 OR RO5541267 OR PD-1 OR PD- L1) AND trial". Two authors independently carried out the searches. Studies were eligible only if they fulfilled the following inclusion criteria: (i) clinical trials or real-world studies; (ii) included NSCLC patients who received immunotherapy (antiPD-1, anti-PD-L1, or anti-CTLA-4) (iii) reported hazard ratios (HRs, immunotherapy cohort $v s$ control) for progression-free survival (PFS) and/or overall survival (OS) stratified by smoking history or reported clinical outcome data such as HRs or odds ratios (ORs) of clinical response for smokers $v s$ non-smokers. Studies that failed to meet these inclusion criteria were excluded. We excluded studies if they contained participants who were also included in other studies.

\section{Data Extraction}

The following information were extracted from the included studies: lead author, publication year, study categories (clinical trial or real-world study), study population, therapeutic regimens, line of treatment, histology (non-squamous $\mathrm{v}$ squamous), smoking status (never-smoker $v s$ current or former smoker). For studies comparing clinical outcomes between an immunotherapy cohort (received immunotherapy only or in combination with chemotherapy) and a control cohort (received chemotherapy or placebo), the HRs for OS or PFS together with the $95 \%$ confidence intervals (CIs) were collected for smokers and non-smokers separately. For studies directly comparing clinical outcomes of immunotherapy (immunotherapy only or in combination with chemotherapy) between smokers and non-smokers, indexes of therapeutic efficacy including HRs for OS or PFS and the OR for the objective response rate (ORR) with the corresponding 95\% CIs were collected for further pooled analysis.

\section{Statistical Analysis}

All analyses were performed using Review Manager software (RevMan version 5.4; Oxford, UK). For studies containing data regarding the clinical benefits of immunotherapy $v s$ 
chemotherapy/placebo, HRs (immunotherapy vs control) for smokers and non-smokers were pooled separately, which facilitated indirect comparisons of clinical benefits of immunotherapy between smokers and non-smokers. For studies containing data for direct comparison of the clinical benefits of immunotherapy in smokers vs non-smokers, HRs (smokers vs non-smokers) or ORs for ORR (smokers vs nonsmokers) were accumulated to obtain pooled results for direct comparison. The $\chi^{2}$ test and $\mathrm{I}^{2}$ statistic were applied to assess statistical heterogeneity, with $\mathrm{P}<0.10$ on the $\chi^{2}$ test and an $\mathrm{I}^{2}$ value $>50 \%$ indicating the existence of heterogeneity. A random model was used to calculate the cumulative HRs, ORs, and their estimated 95\% CIs when heterogeneity was observed among studies; otherwise, a fixed effect model was applied. Null hypothesis (the difference of immunotherapy effect between smokers and not smokers is zero) was tested by comparing the HR among with that of non-smokers following approaches: first, for each trial, an interaction trial-specific HR from the ratio of the reported HRs in smokers and non-smokers patients have to be calculated; second, these trial-specific interaction HRs across trials have to be combined using a random-effects model (15). For the pooled analysis of HRs (immunotherapy $v s$ control) in smokers and non-smokers, subgroup analyses were performed for groups that received immunotherapy as first-line versus second/third-line treatment, as well as for the subgroup of non-squamous NSCLC patients. We did not perform a subgroup analysis for squamous NSCLC patients due to insufficient data. Publication bias was examined by funnel plot and Egger's test. All CIs had two-sided probability coverage of $95 \%$, and a P-value less than 0.05 was considered significant for all statistical tests.

\section{RESULTS}

\section{Characteristics of Included Studies}

We screened 4,654 studies and identified a total of 23 clinical trials and 7 real-world studies that were eligible for inclusion in the present study (Figure 1). Sixteen phase-3 clinical trials involving 10,643 patients compared the observed clinical benefit between an immunotherapy cohort and a chemotherapy/placebo cohort and provided data for the smoking status of patients, and thus, facilitated an indirect comparison of the clinical benefit achieved in smokers versus non-smokers (Table 1). Patients in the immunotherapy cohort received immunotherapy as monotherapy, a combination of two types of immunotherapies, or immunotherapy combined with chemotherapy. The control cohort received chemotherapy as the control treatment in all of the clinical trials except one, in which placebo was administered. We also conducted a pooled analysis for direct comparison of the clinical benefit from immunotherapy between smokers and non-smokers with the data provided by 7 clinical trials and 7 real-world studies, which included 4,777 cases in total (Table 2). In the included studies, the most commonly applied immunotherapy was anti-PD-1 treatment, but some patients received anti-PD-L1 or anti-CTLA-4 therapy in selected studies.
The basic information of all the included studies is summarized in Tables 1, 2.

\section{OS or PFS Benefit From Immunotherapy in Smokers and Non-Smokers}

Of the 10,643 patients included in the 16 studies evaluating the clinical benefits of immunotherapy as compared to chemotherapy/placebo, 5,749 (54.01\%) cases were randomized to the immunotherapy cohort. A total of 9,027 patients were identified as current smokers or former smokers, accounting for $82.36 \%$ of the study population. Compared to chemotherapy/ placebo, immunotherapy significantly prolonged the OS of smokers (HR, 0.76; 95\% CI, 0.69-0.83; $\mathrm{P}<0.001$, Figure 2B) but demonstrated no significant $\mathrm{OS}$ benefit among non-smokers (HR, 0.91; 95\% CI, 0.78-1.06; $\mathrm{P}=0.25$, Figure 2A). With respect to PFS, the HRs were similar in both cohorts, although clinical significance was achieved in smokers (HR, 0.65; 95\% CI, 0.56$0.75 ; \mathrm{P}<0.001$, Figure 2D) but not among non-smokers (HR, 0.68 ; $95 \%$ CI, 0.45-1.03; P=0.07, Figure 2C). Notably, the lack of statistical significance among non-smokers could be attributed to the smaller sample size, and the result does not necessarily prove that immunotherapy is less effective among non-smokers than smokers.

To avoid the risk of ecological bias, we further tested the difference in immunotherapy effectiveness between smokers and non-smokers by comparing the HRs for immunotherapy vs chemo/placebo in the two groups (Figure 3). We found that the HR for OS benefit from immunotherapy $v s$ chemo/placebo in smokers was smaller than that in non-smokers, although the difference was not statistically significant $(\mathrm{P}=0.10)$, which could be attributed to high heterogeneity among studies $\left(\mathrm{I}^{2}=99 \%\right)$. The HR for PFS benefit from immunotherapy vs chemo/placebo among smokers was similar to that in non-smokers (mean difference: $\left.-0.05[-0.37,0.27], \mathrm{P}=0.31, \mathrm{I}^{2}=100 \%\right)$.

\section{Subgroup Analyses}

We also carried out subgroup analyses indirectly comparing outcomes in subgroups with specific histological subtypes or receiving immunotherapy as first- versus second/third-line treatment. A total six trials containing data specific to nonsquamous lung cancer were pooled to evaluated the impact of smoking on the clinical benefit of immunotherapy $v s$ chemotherapy for these patients. As shown in Figure 4, smokers with non-squamous lung cancer experienced significantly prolonged OS ( $\mathrm{HR}, 0.71 ; 95 \% \mathrm{CI}, 0.61-0.84$; $\mathrm{P}<0.0001$, Figure 4B) and PFS (HR, 0.59; 95\% CI, 0.53-0.67; $\mathrm{P}<0.0001$, Figure 4D) after receiving immunotherapy as compared to chemotherapy. Immunotherapy could also significantly improve PFS in non-smokers with nonsquamous lung cancer $(\mathrm{HR}, 0.55 ; 95 \% \mathrm{CI}, 0.33-0.92 ; \mathrm{P}=0.02$, Figure 4C), although the improvement in OS was not significant ( $\mathrm{HR}, 0.68 ; 95 \% \mathrm{CI}, 0.45-1.03 ; \mathrm{P}=0.15$, Figure 4A). We did not carry out this subgroup analysis for patients with squamous cell lung cancer due to the insufficient number of studies available. 


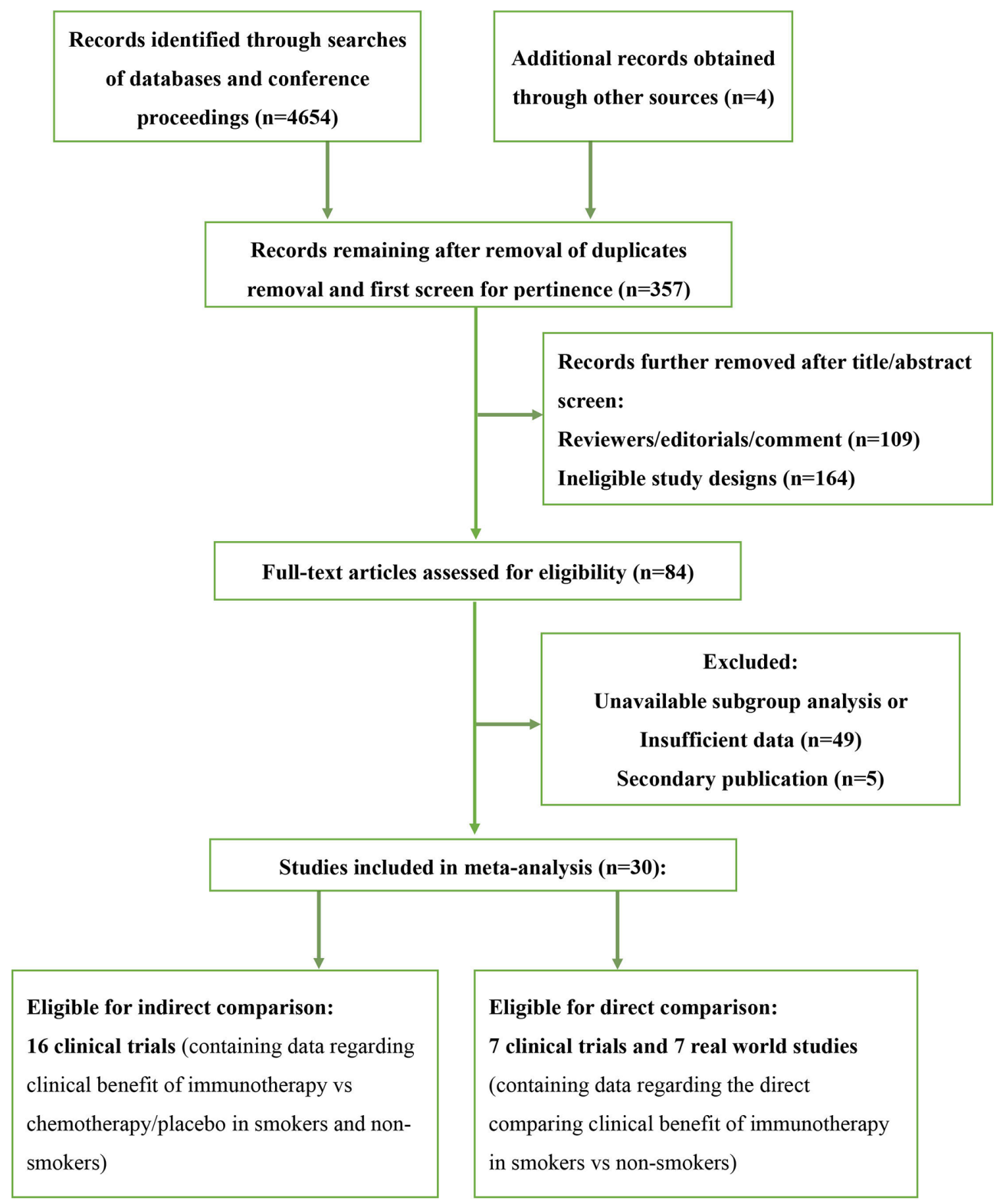

FIGURE 1 | Flow chart of literature search strategy and study selection.

Of all the clinical trials included in our indirect comparison, 11 trials administered immunotherapy (alone or as combination therapy) as the first-line treatment, whereas the remaining 5 studies administered immunotherapy or chemotherapy as secondor third-line treatment. Pooled analysis of the data from these 11 trials involving 7,610 treatment-naive patients showed that the OS (HR, 0.76; 95\% CI, 0.66-0.87; $\mathrm{P}<0.0001$, Figure 5B) and PFS (HR, 0.65 ; 95\% CI, 0.55-0.78; $\mathrm{P}<0.00001$, Figure 5D) were significantly prolonged among smokers who received immunotherapy as firstline treatment compared to those received chemotherapy/placebo as the first-line treatment, whereas no significant improvements in OS
(HR, 0.94; 95\% CI, 0.76-1.15; $\mathrm{P}=0.54$, Figure 5A) and PFS (HR, 0.64 ; 95\% CI, 0.38-1.09; P=0.10, Figure 5C) with immunotherapy were observed for non-smokers. Similarly, the clinical benefits of immunotherapy versus chemotherapy as second- or third-line treatment in terms of prolonged OS and PFS were significant only among smokers (HR for OS, 0.75 [0.67-0.83], $\mathrm{P}<0.00001$, Figure 6B; HR for PFS, 0.61 [0.44-0.86]; $\mathrm{P}=0.005$, Figure 6D) and not among non-smokers (HR for OS, 0.92 [0.63-1.36], $\mathrm{P}=0.69$, Figure 6A; HR for PFS, 0.87 [0.60-1.27]; $\mathrm{P}=0.47$, Figure 6C). Importantly though, the insignificant results for non-smokers may be due to the small sample size of this group. 


\begin{tabular}{|c|c|c|c|c|c|c|c|c|}
\hline Study & Trial type (name) & Population & Immuno therapy & $\begin{array}{l}\text { Line of } \\
\text { treatment }\end{array}$ & Study design & Sample size, $n$ & Squamous, $\mathbf{n}$ & Non-squamous, $n$ \\
\hline Rittmeyer 2016 (16) & Phase 3 (OAK) & NSCLC & Anti-PD-1 & $2-3$ & Atezolizumab vs docetaxel & 850 & 222 & 628 \\
\hline Zhou 2019 (17) & Phase 3 & Non-squamous & Anti-PD-1 & 1 & Camrelizumab + chemo vs chemo & 412 & 0 & 412 \\
\hline Carbone 2017 (18) & Phase 3 (CheckMate 026) & NSCLC & Anti-PD-1 & 1 & Nivolumab vs platinum-based chemo & 541 & 130 & 411 \\
\hline Barlesi 2018 (19) & Phase 3 (JAVELIN Lung 200) & NSCLC & Anti-PD-L1 & $2-3$ & Avelumab vs docetaxel & 792 & 242 & 550 \\
\hline Barlesi 2018-2 (20) & Phase 3 (IMpower132) & Non-squamous & Anti-PD-1 & 1 & Atezolizumab + chemo vs chemo & 578 & 0 & 578 \\
\hline Borghaei 2015 (21) & Phase 3 (CheckMate 057) & Non-squamous & Anti-PD-1 & $2-3$ & Nivolumab vs Docetaxel & 582 & 0 & 582 \\
\hline West 2019 (22) & Phase 3 (IMpower130) & Non-squamous & Anti-PD-1 & 1 & Atezolizumab + chemo vs chemo & 723 & 0 & 723 \\
\hline Gandhi 2018 (23) & Phase 3 (KEYNOTE-189) & Non-squamous & Anti-PD-1 & 1 & Pembrolizumab + chemo vs chemo & 616 & NA & 592 \\
\hline Paz-Ares 2019 (24) & Phase 3 (CheckMate 227 Part 2) & NSCLC & Anti-PD-1 & 1 & Nivolumab + chemo vs chemo & 755 & 212 & 543 \\
\hline Hellmann 2019 (25) & Phase 3 (CheckMate 227) & NSCLC & Anti-PD-1 + anti-CTLA-4 & 1 & Nivolumab + ipilimumab vs chemo & 1166 & 325 & 840 \\
\hline Reck 2016 (26) & Phase 3 (KEYNOTE-024) & NSCLC & Anti-PD-1 & $2-3$ & Pembrolizumab vs platinum-based chemo & 305 & 56 & 249 \\
\hline Reck $2020(27)$ & Phase 3 (CheckMate-9LA) & NSCLC & Anti-PD-1 + anti-CTLA-4 & 1 & Nivolumab + ipililumab + chemo vs chemo & 719 & 62 & 138 \\
\hline Govindan 2017 (28) & Phase 3 (NCT01285609) & Squamous & Anti-CTLA-4 & 1 & Ipilimumab +chemo vs chemo & 749 & 749 & 0 \\
\hline Jotte 2020 (29) & Phase 3 (IMpower131) & Squamous & Anti-PD-1 & 1 & Atezolizumab + chemo vs chemo & 683 & 683 & 0 \\
\hline Antonia 2017 (30) & Phase 3 (PACIFIC) & NSCLC & Anti-PD-L1 & 1 & Durvalumab vs placebo & 713 & 326 & 387 \\
\hline Wu 2019 (31) & Phase 3 (CheckMate-078) & NSCLC & Anti-PD-1 & $2-3$ & Nivolumab vs Docetaxel & 504 & 200 & 304 \\
\hline
\end{tabular}

TABLE 2 | Summary of the 7 clinical trials and 7 real-world studies eligible that provided data for direct comparison.

\begin{tabular}{|c|c|c|c|c|c|c|c|c|}
\hline Studies & Trial/Real-world data & Population & Immuno-therapy & Treatment regimen & Line of treatment & Sample size, $n$ & Squamous, $n$ & Non-squamous, $n$ \\
\hline Gulley 2017 (13) & Phase $1 \mathrm{~b}$ trial & NSCLC & Anti-PD-L1 & avelumab & $2+$ & 184 & 53 & 114 \\
\hline Nishio 2016 (32) & Phase 2 trial (JapicCTI-132073) & Non-squamous NSCLC & Anti-PD-1 & Nivolumab & $2+$ & 76 & 0 & 76 \\
\hline Nishio 2018 (14) & Phase 1b trial (KEYNOTE-025) & NSCLC & Anti-PD-1 & Pembrolizumab & $2+$ & 38 & 6 & 29 \\
\hline Durm 2020 (33) & Phase 2 trial & NSCLC & Anti-PD-1 & Pembrolizumab & $2+$ & 92 & 41 & 51 \\
\hline Goldberg 2020 (34) & Phase 2 trial & NSCLC & Anti-PD-1 & pembrolizumab & $1+$ & 42 & 4 & 36 \\
\hline Feng 2017 (35) & Phase 2 trial & NSCLC & Anti-PD-1 & Nivolumab & $2+$ & 648 & 293 & 354 \\
\hline Marina 2018 (36) & Phase 2 trial (ATLANTIC) & NSCLC & Anti-PD-L1 & Durvalumab & $3+$ & 239 & 51 & 188 \\
\hline Kobayashi 2018 (37) & Real world data & NSCLC & Anti-PD-1 & Nivolumab & $2+$ & 142 & 41 & 83 \\
\hline Lin 2018 (38) & Real-world data & NSCLC & Anti-PD-1 & Nivolumab/pembrolizumab & $1+$ & 71 & 14 & 48 \\
\hline Khozin 2019 (39) & Real-world data & NSCLC & Anti-PD-1 & Nivolumab/pembrolizumab & $1+$ & 1344 & 427 & 872 \\
\hline Barlesi 2020 (40) & Real-world data & NSCLC & Anti-PD-1 & Nivolumab & $1+$ & 1420 & 982 & 438 \\
\hline Chen 2020 (41) & Real-world data & NSCLC & Anti-PD-1 & Nivolumab/pembrolizumab & 2 & 97 & 39 & 58 \\
\hline Morita 2020 (42) & Real-world data & NSCLC & Anti-PD-1 & Nivolumab & $1+$ & 901 & 221 & 610 \\
\hline Weis 2019 (43) & Real-world data & NSCLC & Anti-PD-1/PD-L1 & Nivolumab/atezolizumab & 2 & 124 & 38 & 78 \\
\hline
\end{tabular}




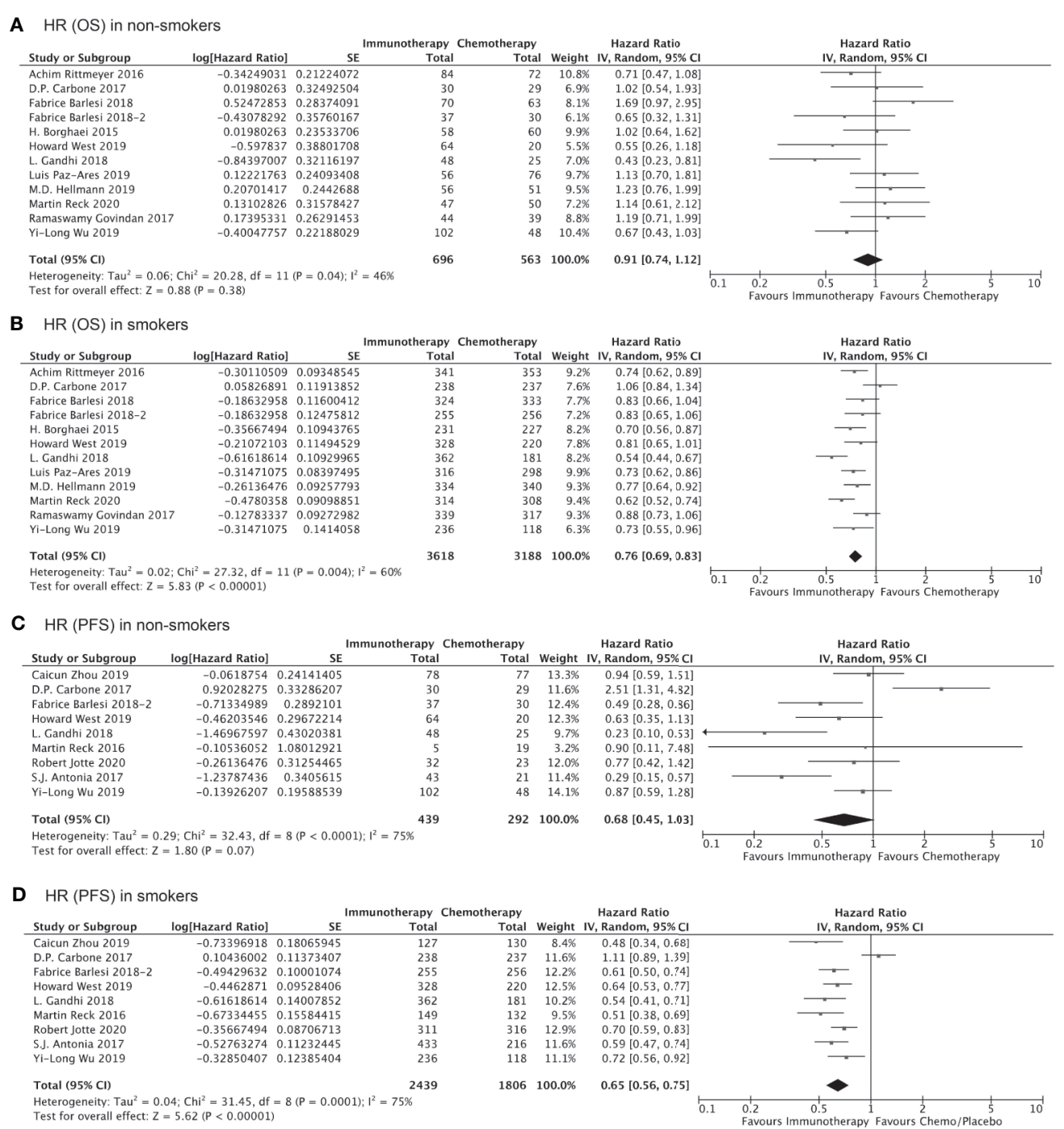

FIGURE 2 | OS or PFS benefits of immunotherapy for NSCLC among smokers and non-smokers.

\section{Direct Comparison of Therapeutic Outcomes Between Smokers and Non-Smokers}

Of the 4,777 patients included in 9 clinical trials and 7 real-world studies that directly compared the clinical outcomes of anti-PD1/PD-L1 immunotherapy (monotherapy or combined with chemotherapy) between smoker and non-smokers with NSCLC, 3,098 (64.85\%) patients were current or former smokers. There was no observed publication bias according to Egger's test (Figure 7). The pooled analysis demonstrated that smokers achieved better OS (HR, 0.86; 95\% CI, 0.75-0.99; $\mathrm{P}=0.04$, Figure 8A) and PFS (HR, 0.69; 95\% CI, 0.60-0.81; $\mathrm{P}<0.00001$, Figure 8B) compared with non-smokers after receiving immunotherapy. Smokers also tended to achieve a higher ORR than non-smokers, although the difference was not statistically significant in the pooled analysis (OR, 1.20; 95\% CI, 0.94-1.53; $\mathrm{P}=0.15$, Figure 8C). We did not perform subgroup analyses for direct comparisons due to insufficient data availability.

\section{DISCUSSION}

As a well-known risk factor for lung cancer, smoking history plays a role in oncogenesis by altering tumor cells directly or by influencing the tumor microenvironment $(44,45)$. However, the potential effect of smoking history on the clinical outcomes of immunotherapy remains to be confirmed. This meta-analysis in which all the available data from clinical trials and real-world studies were pooled provides a comprehensive and systematic review of the impact of smoking history on the therapeutic outcomes of immunotherapy in NSCLC patients. We found that the survival benefit of immunotherapy over chemotherapy/placebo was more significant among smokers than among non-smokers, independent 
A Comparison of HR (OS) between smokers and non-smokers

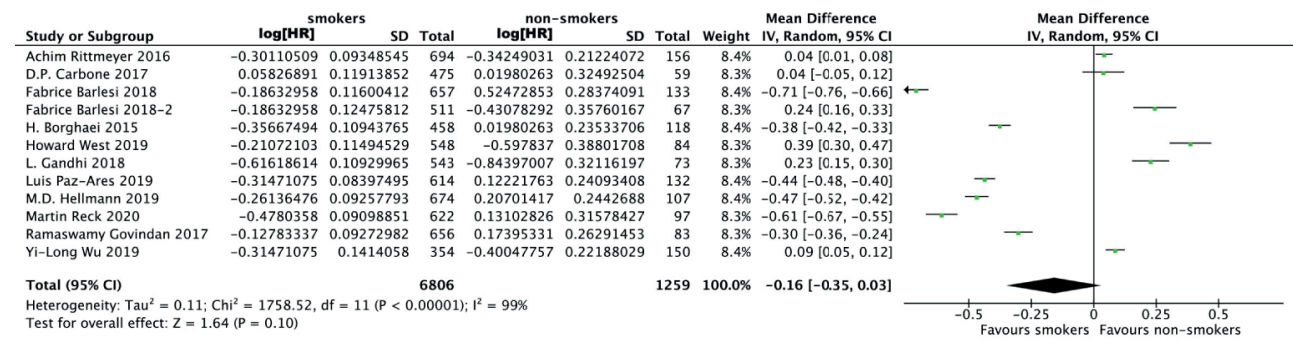

\section{B Comparison of HR (PFS) between smokers and non-smokers}

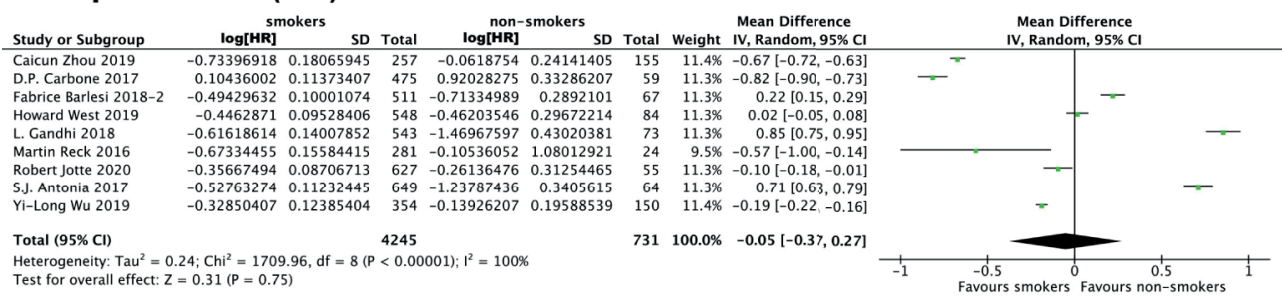

FIGURE 3 | Comparison of Hazard Ratio regarding overall survival benefit (A) or progression free survival benefit (B) of immunotherapy vs chemo/placebo between smokers and non-smokers.

A $\operatorname{HR}(\mathrm{OS})$ in non-smokers

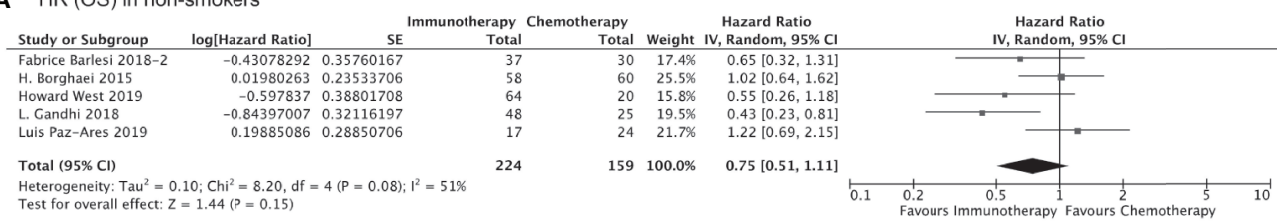

B $\mathrm{HR}(\mathrm{OS})$ in smokers

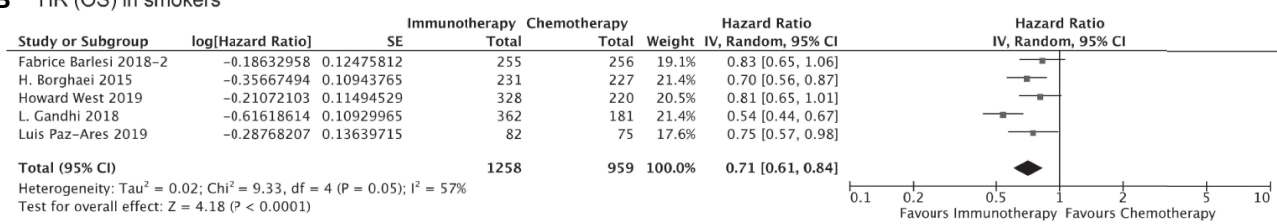

C $\mathrm{HR}(\mathrm{PFS})$ in non-smokers

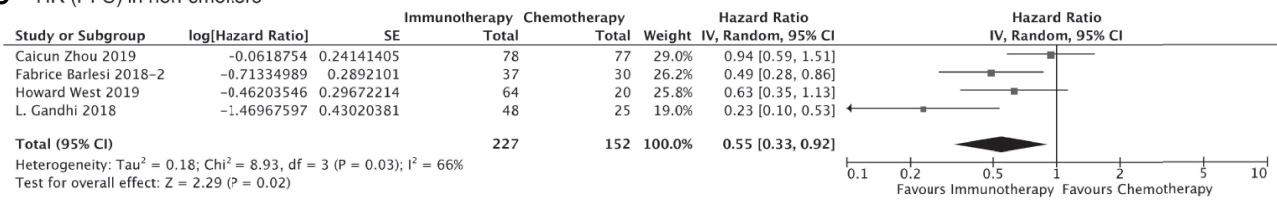

D $\quad \mathrm{HR}(\mathrm{PFS})$ in smokers

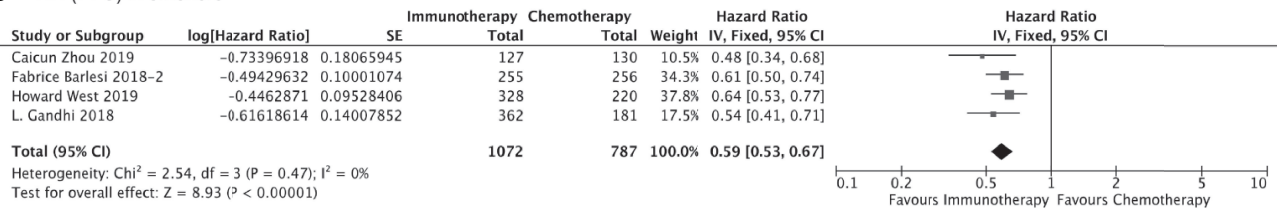

FIGURE 4 | OS or PFS benefits from immunotherapy for non-squamous NSCLC among smokers and non-smokers. 

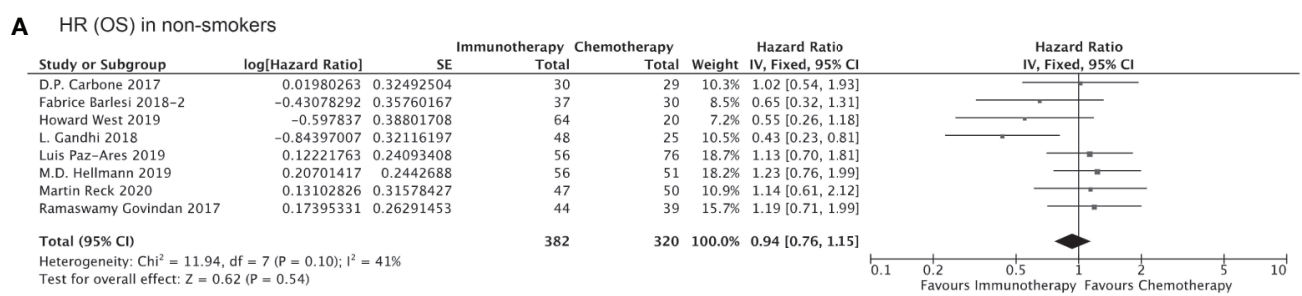

B $\quad H R(O S)$ in smokers

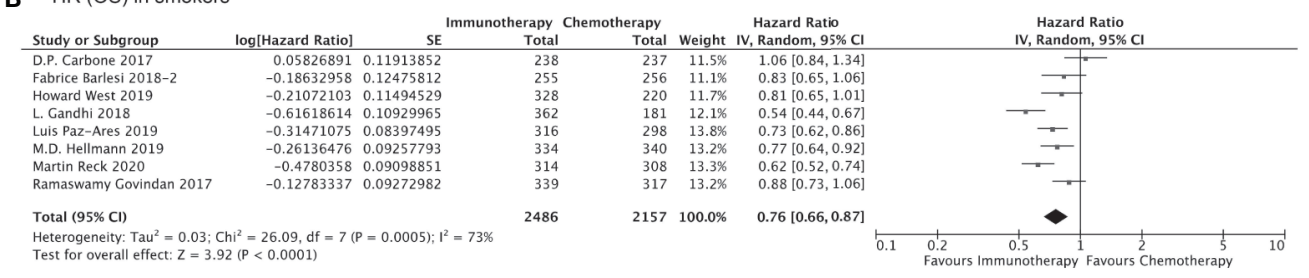

C $\quad H R$ (PFS) in non-smokers

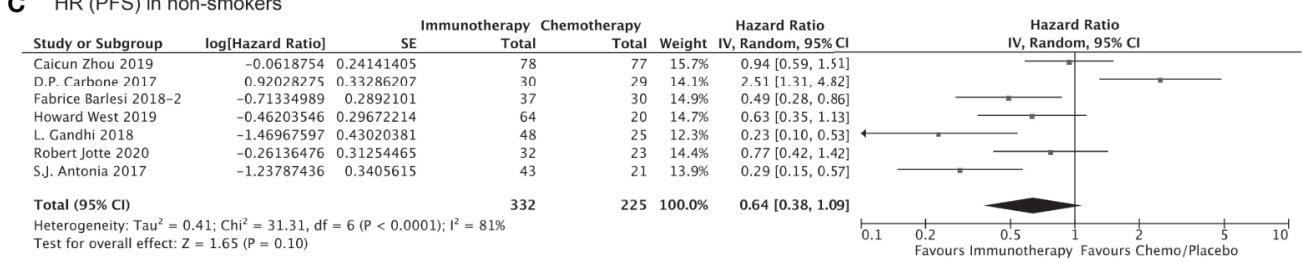

D $\mathrm{HR}$ (PFS) in smokers

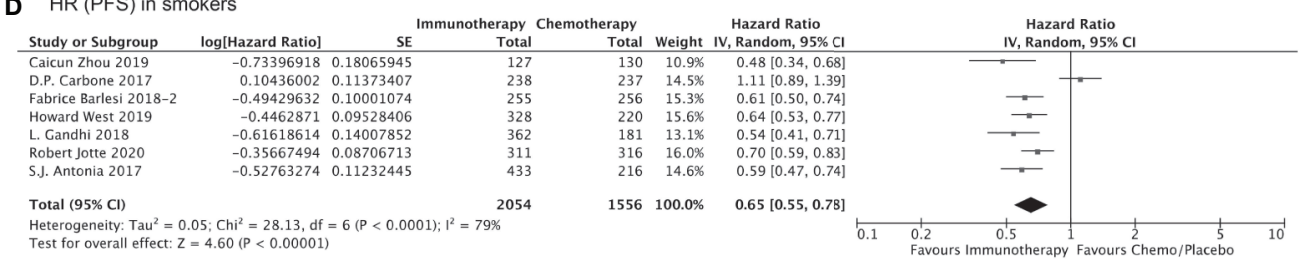

FIGURE 5 | OS or PFS benefits from immunotherapy as first-line treatment among smokers and non-smokers.

of the histologic subtype or line of treatment. Also, patients with a smoking history benefited more from immunotherapy than did non-smokers. The present meta-analysis enhances the evidence that smoking history, as a clinical parameter, can be applied as a predictor of immunotherapy efficacy and guide patient's selection for immunotherapy among NSCLC patients.

Since the ground-breaking discovery of checkpoint blockade through anti-PD-1- and anti-PD-L1-based immunotherapy and the successful application of these immunotherapies in lung cancer, melanoma, etc., continuous efforts have focused on methods for identifying the patients most likely to respond to immunotherapy. Even though lung cancer is one of the cancer types that respond best to immunotherapy, only $20 \sim 30 \%$ of patients have been found to actually benefit from immunotherapy (5). PD-1 and PD-L1, as the direct targets of checkpoint blockade, have been widely applied as biomarkers in attempts to predict the potential response to immunotherapy (38). However, accumulating evidence indicates that the expression status of PD-1 or PD-L1 alone is insufficient to determine which patients should be offered PD-1- or PD-L1targeting therapy (46). A tremendous amount of work has focused on the development of complicated predictive algorithms integrating genomic and tumor microenvironment features that can identify patients who are likely to benefit from immunotherapy (68), but little attention has been paid to clinical parameters that might also facilitate responder identification. Several studies have aimed to evaluate the significance of smoking history on the clinical outcomes of immunotherapy, with inconsistent findings and some studies reporting no significant relevance, which in no small part, can be attributed to the modest sample sizes of the study populations (7-10). Pooled analyses of the previously available data involving a large number of patients can provide much more solid conclusions regarding this issue. A review of the literature published through 2019 investigated the role of tobacco smoking in immunotherapy targeting PD-1/PD-L1 by summarizing the findings of clinical trials and reached the conclusion that NSCLC in current/former smokers responded better than that in nonsmokers to immunotherapies (11). However, the conclusion was derived from a descriptive summary of previous findings, and no statistical evidence was derived from pooled analyses. Also, only a limited number of studies were included in that previous review, which restricted the feasibility of pooled analyses. With increasing numbers of relevant clinical trials as well as real-world studies published recent years, the now abundant data facilitated the pooled analyses in the current study. We evaluated the impact of smoking 
A $\quad H R(O S)$ in non-smokers

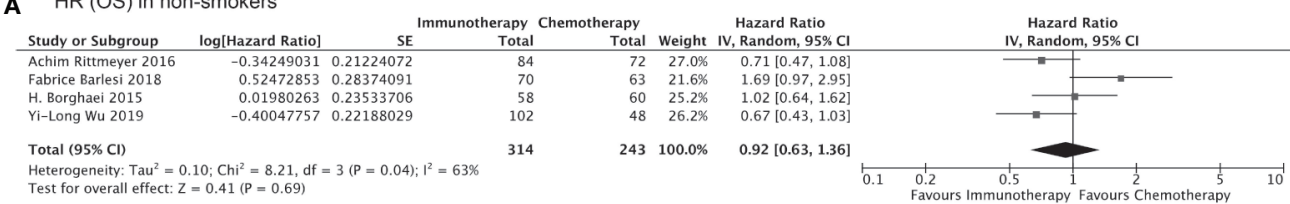

B $\quad H R(O S)$ in smokers

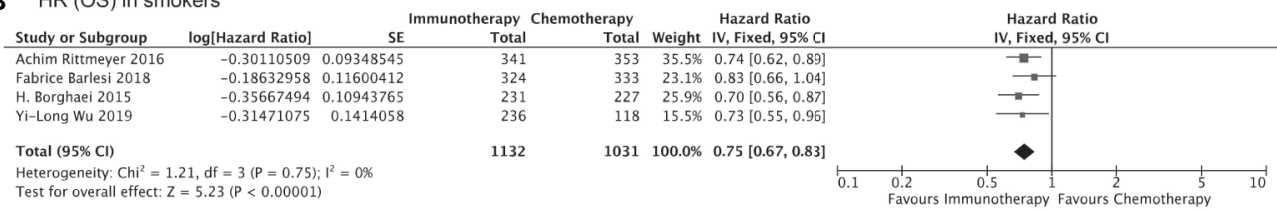

C $\quad \mathrm{HR}(\mathrm{PFS})$ in non-smokers
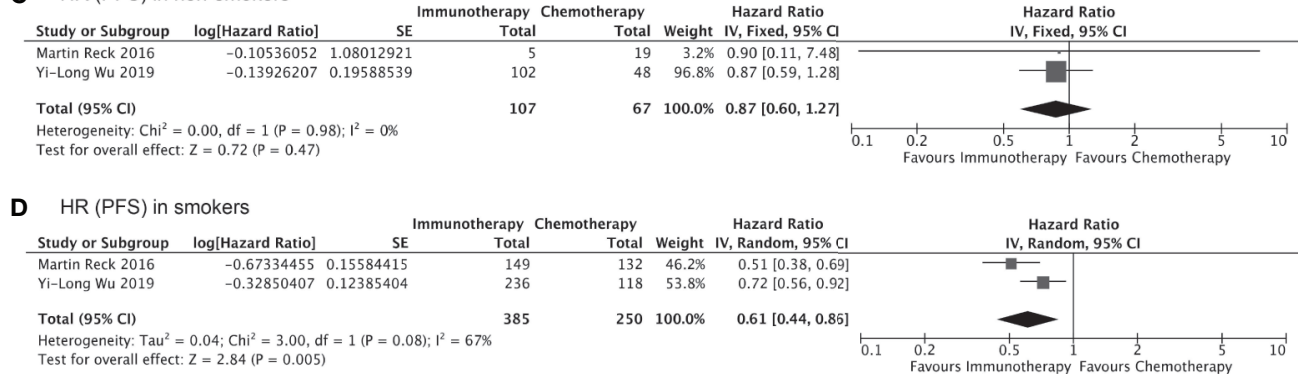

FIGURE 6 | OS or PFS benefits from immunotherapy as second- or third-line treatment among smokers and non-smokers.

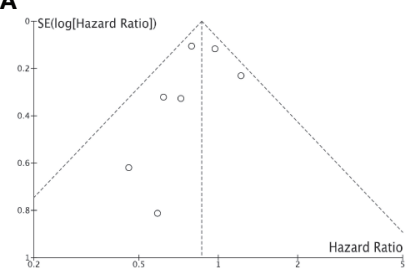

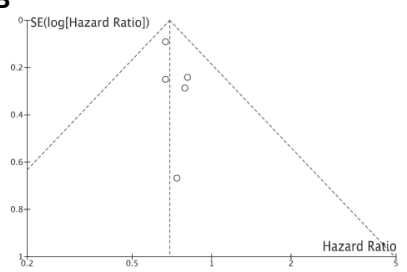

C

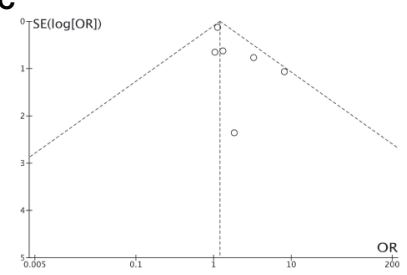

FIGURE 7 | Funnel plot for publication bias of the direct comparison of HR-OS (A), HR-PFS (B) and ORR (C) between non-smokers and (C) smokers.

history on the outcomes of immunotherapy by demonstrating the survival gains with immunotherapy versus chemotherapy/placebo in smokers and non-smokers separately and also by directly comparing the clinical outcomes of immunotherapy between smokers and non-smokers. All of the different analyses in our study support the conclusion that immunotherapy offers better survival benefits in smokers compared with non-smokers. Another recently published study also performed meta-analysis to evaluate the impact of smoking on the therapeutic outcome of immunotherapy (12). Although the conclusions are similar, our study still outperforms the published one in terms of: a much larger sample size with several newly published clinical trials included; the impact of smoking status on the therapeutic efficacy of immunotherapy was evaluated not only by indirect comparison (evaluating the clinical benefit of immunotherapy in smokers and non-smokers separately), but also by direct comparison (comparing the therapeutic outcome of smokers with that of non-smokers after immunotherapy); systematic subgroup analysis was carried out to preclude potential confounding effects.

Importantly, although the survival improvement achieved with immunotherapy among non-smokers was not as significant as that among smokers, we cannot conclude that smokers benefit more from immunotherapy than non-smokers based only on the results of this analysis. Because the sample size of non-smokers analyzed in our study was far smaller than that of smokers, the statistical insignificance in non-smokers could be attributed to the small sample size. To better assess the differences in pooled OS-HRs and PFS-HRs for smokers and non-smokers, we further carried out an interaction test, which showed a relatively improved HR for smokers but without statistical significance. The statistical insignificance in these results could also be attributed to the high heterogeneity among studies included. Overall, the results derived 
A $\operatorname{HR}(\mathrm{OS})$

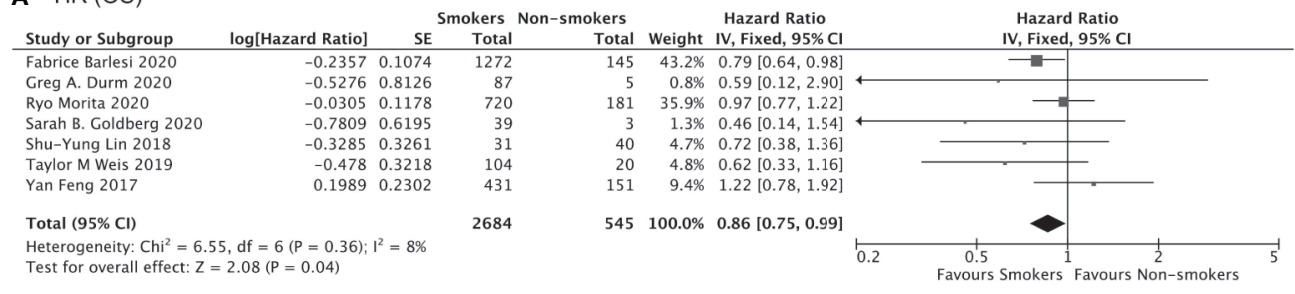

B $\mathrm{HR}$ (PFS)

\begin{tabular}{|c|c|c|c|c|c|c|c|c|c|c|}
\hline Study or Subgroup & log[Hazard Ratio] & \multicolumn{2}{|c|}{ Smokers } & \multicolumn{2}{|l|}{ Non-smokers } & $\begin{array}{l}\text { Hazard Ratio } \\
\text { IV, Fixed, 95\% CI }\end{array}$ & \multicolumn{4}{|c|}{$\begin{array}{c}\text { Hazard Ratio } \\
\text { IV, Fixed, } 95 \% \mathrm{CI}\end{array}$} \\
\hline Greg A. Durm 2020 & -0.3011 & 0.6675 & 87 & 5 & $1.3 \%$ & $0.74[0.20,2.74]$ & & & & \\
\hline Minjiang Chen 2020 & -0.207 & 0.2419 & 57 & 40 & $10.2 \%$ & $0.81[0.51,1.31]$ & & & - & \\
\hline Ryo Morita 2020 & -0.4005 & 0.0915 & 720 & 181 & $71.6 \%$ & $0.67[0.56,0.80]$ & & & & \\
\hline Shu-Yung Lin 2018 & -0.4005 & 0.2506 & 31 & 40 & $9.5 \%$ & $0.67[0.41,1.09]$ & & & - & \\
\hline Taylor M Weis 2019 & -0.2357 & 0.2871 & 104 & 20 & $7.3 \%$ & $0.79[0.45,1.39]$ & & & & \\
\hline Total $(95 \% \mathrm{Cl})$ & & & 999 & 286 & $100.0 \%$ & $0.69[0.60,0.81]$ & & & & \\
\hline $\begin{array}{l}\text { Heterogeneity: } \mathrm{Chi}^{2}= \\
\text { Test for overall effect }\end{array}$ & $\begin{array}{l}0.81, d f=4(P=0.9 \\
Z=4.74(P<0.0000\end{array}$ & $\begin{array}{l}\text { 4); } 1^{2}=0 \% \\
01)\end{array}$ & & & & & 0.2 & $\begin{array}{c}0.5 \\
\text { viours Smokers }\end{array}$ & Favours No & $\begin{array}{l}1 \\
\text { lon-smokers }\end{array}$ \\
\hline
\end{tabular}

C ORR

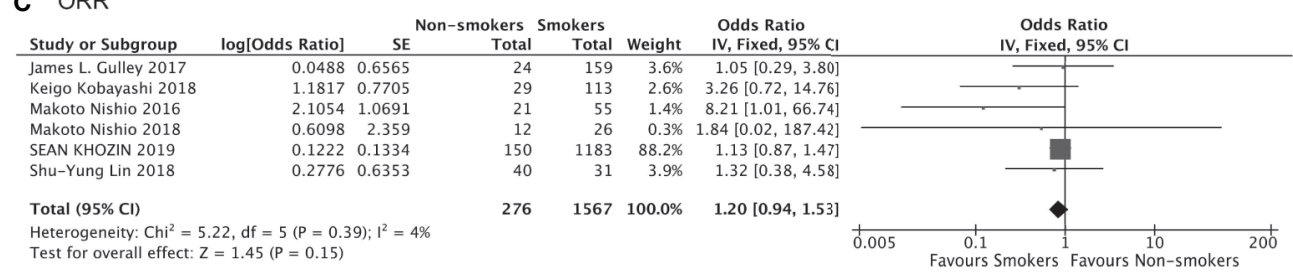

FIGURE 8 | Direct comparison of immunotherapy outcomes between smokers and non-smokers with NSCLC.

from indirectly comparing the immunotherapy benefit between smokers and non-smokers cannot provide solid evidence that immunotherapy works better for smokers. Results derived from a direct comparison will better support the conclusion that smokers may benefit more from immunotherapy as compared to non-smokers.

The next logical question is why does a smoking history contribute to a better response to immunotherapy. The first and most obvious explanation is the correlation between smoking and TMB (47). TMB is another widely accepted biomarker for predicting the immunotherapy response among patients (48). It is widely acknowledged that NSCLC and melanoma are the two cancer types that most benefit from immunotherapy, and this has largely been attributed to the high TBM in both cancer types (49). While ultraviolet exposure is the major cause of DNA damage and elevated TMB in melanoma as a skin cancer, tobacco exposure likely contributes to the high TMB in lung cancer. A previous study showed that lung cancers in smokers had a significantly higher TMB compared with lung cancers in never-smokers (49). A high TMB contributes to the production of a higher abundance of neoantigens, which facilitates the recognition of cancerous cells by the immune system. Also, accumulation of neoantigens on the surface of tumor cells can stimulate the recruitment of cytotoxic immune cells into the tumor microenvironment, which will further boost the therapeutic efficacy of immunotherapy.

Additionally, smoking may exert an impact on the tumor microenvironment in a manner beyond TMB. In some inflammatory pulmonary disorders, such as emphysema and chronic obstructive pulmonary disease, smoking is thought to play a role in skewing the local immune microenvironment to a proinflammatory phenotype (50). Previous studies have also reported that the immunologic homeostasis within the tumor microenvironment is less compromised in never-smokers compared with ever-smokers $(51,52)$. It is believed that immune cells are recruited in response to tobacco exposure, in an attempt to minimize the damage induced by the carcinogenic substance via a pro-inflammatory reaction (53). However, the immune cells could also partially contribute to the harmful tumor microenvironment that promotes tumor growth (54). Smoking can influence the tumor microenvironment not only during the stage of tumor initiation, but may continue its effect throughout the process of tumor progression. For example, tobacco exposure was reported to polarize macrophages to a proinflammatory phenotype, M1 (55). Macrophages, as the major component within the tumor microenvironment, dictate the recruitment of other immune cells based on their functional status, with the immune suppressive macrophage phenotype suppressing T-cell infiltration and the proinflammatory macrophage phenotype efficiently promoting immune cell recruitment (56). Immune cell infiltration, and specifically infiltration of $\mathrm{T}$ cells, is believed to be a perquisite for immunotherapy to exert a therapeutic effect (57). As a substitute for direct examination of tumor tissue obtained by biopsy, smoking history might also convey implicit information regarding the status of the tumor immune microenvironment.

Smoking status was long ago found to correlate with certain oncogene mutations in lung cancer, with mutation of oncogenes 
such as KRAS commonly found in smokers (58). On the other hand, mutations of EGFR, ROS1, and EML4-ALK fusion occur more frequently in non-smokers (51). Accumulating studies have proven that lung cancers harboring oncogenic alterations in ROS1 or EML4-ALK respond poorly to immunotherapy (59), whereas KRAS mutations are associated with an improved response to immunotherapy (60). Differences in the oncogene mutation spectrum may contribute to the differences in therapeutic outcome of immunotherapy among smokers and non-smokers. Furthermore, previous research has suggested that NSCLC in smokers demonstrates significantly higher expression of PD-L1 (61), which could also contribute to the predictive value of smoking for the response to immunotherapy.

Even with the comprehensive nature of our findings, the limitations of the present study need to be considered. First, as a meta-analysis carried out by pooling the published data, analysis of data from individual patients was not possible in our study. Thus, we could not control the test cohort and control cohort to make then comparable across all the studies. Differences in study design, therapeutic dosages, tumor stages, and patients' performance status could be potential confounding factors. Secondly, significant heterogeneity among the included studies was observed in the analysis of OS and especially PFS. Heterogeneity among studies might be attributed to differences in study design, therapeutic regimens, tumor stages, follow-up system and the definition of disease progression across different study centers. Thirdly, we did not carry out subgroup analyses according to tumor stage, oncogenic alteration (e.g., EGFR mutation, EML4-ALK fusion, KRAS mutation), expression status of PD-1/PD-L1, etc. due to the limitations of the data. Last but not least, the study design of real-world studies may have been less well-organized as compared to that of clinical trials, which would compromise the quality of the reported data and thus might compromise the evidence level.

\section{CONCLUSIONS}

In conclusion, our study systematically evaluated the impact of smoking history on the therapeutic response to immunotherapy

\section{REFERENCES}

1. Bray F, Ferlay J, Soerjomataram I, Siegel RL, Torre LA, Jemal A. Global Cancer Statistics 2018: GLOBOCAN Estimates of Incidence and Mortality Worldwide for 36 Cancers in 185 Countries. CA Cancer J Clin (2018) 68:394424. doi: $10.3322 /$ caac. 21492

2. Ettinger DS, Wood DE, Aggarwal C, Aisner DL, Akerley W, Bauman JR, et al. Nccn Guidelines Insights: Non-Small Cell Lung Cancer, Version 1.2020. J Natl Compr Canc Netw (2019) 17:1464-72. doi: 10.6004/jnccn.2019.0059

3. Topalian SL, Hodi FS, Brahmer JR, Gettinger SN, Smith DC, McDermott DF, et al. Safety, Activity, and Immune Correlates of Anti-PD-1 Antibody in Cancer. N Engl J Med (2012) 366:2443-54. doi: 10.1056/NEJMoa1200690

4. Brahmer JR, Govindan R, Anders RA, Antonia SJ, Sagorsky S, Davies MJ, et al. The Society for Immunotherapy of Cancer Consensus Statement on Immunotherapy for the Treatment of Non-Small Cell Lung Cancer (NSCLC). J Immunother Cancer (2018) 6:75. doi: 10.1186/s40425-018-0382-2

5. Vansteenkiste JF. Immunotherapy in Lung Cancer. ESMO Open (2018) 3: e000311. doi: 10.1136/esmoopen-2017-000311 in terms of survival benefit and ORR. We demonstrated that patients with a smoking history achieved longer survival times than non-smokers after receiving immunotherapy, through comparisons of the survival gains with immunotherapy versus chemotherapy/placebo between smokers and non-smokers, and by pooling the direct comparisons of survival gain and response rate with immunotherapy between smokers and nonsmokers. Smoking history could be a simple index that guide the selection of potential responders to immunotherapy among NSCLC patients.

\section{DATA AVAILABILITY STATEMENT}

The raw data supporting the conclusions of this article will be made available by the authors, without undue reservation.

\section{AUTHOR CONTRIBUTIONS}

WZ, WJ, HW, and JH conceived and designed research. WZ, WJ, and QY collected data and conducted research. WZ, WJ, and CS analyzed and interpreted data. WZ and WJ wrote the initial paper. HW and JH revised the paper. QY had primary responsibility for final content. All authors contributed to the article and approved the submitted version.

\section{FUNDING}

The present study was supported by grants from the Guangxi Medical and Health Appropriate Technology Development and Promotion Application Project (grant no. S2019049), Clinical Research Specific Funding Project of Wu Jieping Medical Foundation (grant no. 320.6750.19088-65) and Medical Research Funding Project of Beijing Medical and Health Public Welfare Foundation (grant no. TA201901).

6. Incorvaia L, Fanale D, Badalamenti G, Barraco N, Bono M, Corsini LR, et al. Programmed Death Ligand 1 (PD-L1) as a Predictive Biomarker for Pembrolizumab Therapy in Patients With Advanced non-Small-Cell Lung Cancer (NSCLC). Adv Ther (2019) 36:2600-17. doi: 10.1007/s12325-019-01057-7

7. Owada-Ozaki Y, Muto S, Takagi H, Inoue T, Watanabe Y, Fukuhara M, et al. Prognostic Impact of Tumor Mutation Burden in Patients With Completely Resected Non-Small Cell Lung Cancer: Brief Report. J Thorac Oncol (2018) 13:1217-21. doi: 10.1016/j.jtho.2018.04.003

8. Yu Y, Zeng D, Ou Q, Liu S, Li A, Chen Y, et al. Association of Survival and Immune-Related Biomarkers With Immunotherapy in Patients With Non-Small Cell Lung Cancer: A Meta-Analysis and Individual Patient-Level Analysis. JAMA Netw Open (2019) 2:e196879. doi: 10.1001/jamanetworkopen.2019.6879

9. Lim SM, Choi JW, Hong MH, Jung D, Lee CY, Park SY, et al. Indoor Radon Exposure Increases Tumor Mutation Burden in Never-Smoker Patients With Lung Adenocarcinoma. Lung Cancer (2019) 131:139-46. doi: 10.1016/ j.lungcan.2019.04.002

10. Nagahashi M, Sato S, Yuza K, Shimada Y, Ichikawa H, Watanabe S, et al. Common Driver Mutations and Smoking History Affect Tumor Mutation 
Burden in Lung Adenocarcinoma. J Surg Res (2018) 230:181-5. doi: 10.1016/ j.jss.2018.07.007

11. Norum J, Nieder C. Tobacco Smoking and Cessation and PD-L1 Inhibitors in Non-Small Cell Lung Cancer (NSCLC): A Review of the Literature. ESMO Open (2018) 3:e000406. doi: 10.1136/esmoopen-2018-000406

12. Lee KWC, Lord SJ, Kasherman L, Marschner I, Stockler M, Gralla R, et al. The Impact of Smoking on the Effectiveness of Immune Checkpoint Inhibitors - a Systematic Review and Meta-Analysis. Acta Oncol (2020) 59:96-100. doi: 10.1080/0284186x.2019.1670354

13. Gulley JL, Rajan A, Spigel DR, Iannotti N, Chandler J, Wong DJL, et al. Avelumab for Patients With Previously Treated Metastatic or Recurrent NonSmall-Cell Lung Cancer (JAVELIN Solid Tumor): Dose-Expansion Cohort of a Multicentre, Open-Label, Phase 1b Trial. Lancet Oncol (2017) 18:599-610. doi: 10.1016/s1470-2045(17)30240-1

14. Nishio M, Takahashi T, Yoshioka H, Nakagawa K, Fukuhara T, Yamada K, et al. KEYNOTE-025: Phase 1b Study of Pembrolizumab in Japanese Patients With Previously Treated Programmed Death Ligand 1-Positive Advanced Non-SmallCell Lung Cancer. Cancer Sci (2019) 110:1012-20. doi: 10.1111/cas.13932

15. Fisher DJ, Carpenter JR, Morris TP, Freeman SC, Tierney JF. Meta-Analytical Methods to Identify Who Benefits Most From Treatments: Daft, Deluded, or Deft Approach? Bmj (2017) 356:j573. doi: 10.1136/bmj.j573

16. Rittmeyer A, Barlesi F, Waterkamp D, Park K, Ciardiello F, von Pawel J, et al. Atezolizumab Versus Docetaxel in Patients With Previously Treated Non-SmallCell Lung Cancer (OAK): A Phase 3, Open-Label, Multicentre Randomised Controlled Trial. Lancet (2017) 389:255-65. doi: 10.1016/s0140-6736(16)32517-x

17. Zhou C, Chen G, Huang Y, Zhou J, Lin L, Feng J, et al. Camrelizumab Plus Carboplatin and Pemetrexed Versus Chemotherapy Alone in ChemotherapyNaive Patients With Advanced Non-Squamous Non-Small-Cell Lung Cancer (CameL): A Randomised, Open-Label, Multicentre, Phase 3 Trial. Lancet Respir Med (2021) 9:305-14. doi: 10.1016/s2213-2600(20)30365-9

18. Carbone DP, Reck M, Paz-Ares L, Creelan B, Horn L, Steins M, et al. FirstLine Nivolumab in Stage IV or Recurrent Non-Small-Cell Lung Cancer. $N$ Engl J Med (2017) 376:2415-26. doi: 10.1056/NEJMoa1613493

19. Barlesi F, Vansteenkiste J, Spigel D, Ishii H, Garassino M, de Marinis F, et al. Avelumab Versus Docetaxel in Patients With Platinum-Treated Advanced Non-Small-Cell Lung Cancer (JAVELIN Lung 200): An Open-Label, Randomised, Phase 3 Study. Lancet Oncol (2018) 19:1468-79. doi: 10.1016/ s1470-2045(18)30673-9

20. Barlesi F, Nishio M, Cobo M, Steele N, Paramonov V, Parente B, et al. Impower132: Efficacy of Atezolizumab + Carboplatin/Cisplatin + Pemetrexed as $1 \mathrm{~L}$ Treatment in Key Subgroups With Stage IV Non-Squamous NSCLC. ESMO (2018). doi: 10.1093/annonc/mdy 424.066

21. Borghaei H, Paz-Ares L, Horn L, Spigel DR, Steins M, Ready NE, et al. Nivolumab Versus Docetaxel in Advanced Nonsquamous Non-Small-Cell Lung Cancer. N Engl J Med (2015) 373:1627-39. doi: 10.1056/NEJMoa1507643

22. West H, McCleod M, Hussein M, Morabito A, Rittmeyer A, Conter HJ, et al. Atezolizumab in Combination With Carboplatin Plus Nab-Paclitaxel Chemotherapy Compared With Chemotherapy Alone as First-Line Treatment for Metastatic Non-Squamous Non-Small-Cell Lung Cancer (Impower130): A Multicentre, Randomised, Open-Label, Phase 3 Trial. Lancet Oncol (2019) 20:924-37. doi: 10.1016/s1470-2045(19)30167-6

23. Gandhi L, Rodríguez-Abreu D, Gadgeel S, Esteban E, Felip E, De Angelis F, et al. Pembrolizumab Plus Chemotherapy in Metastatic Non-Small-Cell Lung Cancer. N Engl J Med (2018) 378:2078-92. doi: 10.1056/NEJMoa1801005

24. Paz Ares L, Cielanu TE, Yu X, Saman P, Pluzanski A, Nagrial A, et al. Nivolumab + Chemotherapy vs Chemotherapy as First-Line Treatment for Advanced NSCLC: Checkmate 227 Part 2 Final Analysis. ESMO IMMUNO-ONCOL (2019).

25. Hellmann MD, Paz-Ares L, Bernabe Caro R, Zurawski B, Kim SW, Carcereny Costa E, et al. Nivolumab Plus Ipilimumab in Advanced Non-Small-Cell Lung Cancer. N Engl J Med (2019) 381:2020-31. doi: 10.1056/NEJMoa1910231

26. Reck M, Rodríguez-Abreu D, Robinson AG, Hui R, Csőszi T, Fülöp A, et al. Pembrolizumab Versus Chemotherapy for PD-L1-Positive non-Small-Cell Lung Cancer. N Engl J Med (2016) 375:1823-33. doi: 10.1056/NEJMoa1606774

27. Reck M, Ciuleanu TE, Cobo Dols M, Schenker M, Zurawski B, Menezes J, et al. Nivolumab + Ipilimumab +2 Cycles of Platinum-Doublet Chemotherapy vs 4 Cycles Chemotherapy as First-Line Treatment for Stage IV/Recurrent NSCLC: Checkmate 9LA. ASCO (2020). doi: 10.1200/ JCO.2020.38.15_suppl.9501
28. Govindan R, Szczesna A, Ahn MJ, Schneider CP, Gonzalez Mella PF, Barlesi F, et al. Phase III Trial of Ipilimumab Combined With Paclitaxel and Carboplatin in Advanced Squamous Non-Small-Cell Lung Cancer. J Clin Oncol (2017) 35:3449-57. doi: 10.1200/jco.2016.71.7629

29. Jotte R, Cappuzzo F, Vynnychenko I, Stroyakovskiy D, Rodríguez-Abreu D, Hussein M, et al. Atezolizumab in Combination With Carboplatin and NabPaclitaxel in(Impower131): Results From a Randomized Phase III Trial. J Thorac Oncol (2020) 15:1351-60. doi: 10.1016/j.jtho.2020.03.028

30. Antonia SJ, Villegas A, Daniel D, Vicente D, Murakami S, Hui R, et al. Durvalumab After Chemoradiotherapy in Stage III Non-Small-Cell Lung Cancer. N Engl J Med (2017) 377:1919-29. doi: 10.1056/NEJMoa1709937

31. Wu YL, Lu S, Cheng Y, Zhou C, Wang J, Mok T, et al. Nivolumab Versus Docetaxel in a Predominantly Chinese Patient Population With Previously Treated Advanced NSCLC: Checkmate 078 Randomized Phase III Clinical Trial. J Thorac Oncol (2019) 14:867-75. doi: 10.1016/j.jtho.2019.01.006

32. Nishio M, Hida T, Atagi S, Sakai H, Nakagawa K, Takahashi T, et al. Multicentre Phase II Study of Nivolumab in Japanese Patients With Advanced or Recurrent Non-Squamous Non-Small Cell Lung Cancer. ESMO Open (2016) 1:e000108. doi: 10.1136/esmoopen-2016-000108

33. Durm GA, Jabbour SK, Althouse SK, Liu Z, Sadiq AA, Zon RT, et al. A Phase 2 Trial of Consolidation Pembrolizumab Following Concurrent Chemoradiation for Patients With Unresectable Stage III Non-Small Cell Lung Cancer: Hoosier Cancer Research Network LUN 14-179. Cancer (2020) 126:4353-61. doi: $10.1002 / \mathrm{cncr} .33083$

34. Goldberg SB, Schalper KA, Gettinger SN, Mahajan A, Herbst RS, Chiang AC, et al. Pembrolizumab for Management of Patients With NSCLC and Brain Metastases: Long-Term Results and Biomarker Analysis From a NonRandomised, Open-Label, Phase 2 Trial. Lancet Oncol (2020) 21:655-63. doi: 10.1016/s1470-2045(20)30111-x

35. Feng Y, Wang X, Bajaj G, Agrawal S, Bello A, Lestini B, et al. Nivolumab Exposure-Response Analyses of Efficacy and Safety in Previously Treated Squamous or Nonsquamous Non-Small Cell Lung Cancer. Clin Cancer Res (2017) 23:5394-405. doi: 10.1158/1078-0432.Ccr-16-2842

36. Garassino MC, Cho BC, Kim JH, Mazières J, Vansteenkiste J, Lena H, et al. Durvalumab as Third-Line or Later Treatment for Advanced Non-Small-Cell Lung Cancer (ATLANTIC): An Open-Label, Single-Arm, Phase 2 Study. Lancet Oncol (2018) 19:521-36. doi: 10.1016/s1470-2045(18)30144-X

37. Kobayashi K, Nakachi I, Naoki K, Satomi R, Nakamura M, Inoue T, et al. RealWorld Efficacy and Safety of Nivolumab for Advanced Non-Small-Cell Lung Cancer: A Retrospective Multicenter Analysis. Clin Lung Cancer (2018) 19: e349-58. doi: 10.1016/j.cllc.2018.01.001

38. Lin SY, Yang CY, Liao BC, Ho CC, Liao WY, Chen KY, et al. Tumor PD-L1 Expression and Clinical Outcomes in Advanced-Stage Non-Small Cell Lung Cancer Patients Treated With Nivolumab or Pembrolizumab: Real-World Data in Taiwan. J Cancer (2018) 9:1813-20. doi: 10.7150/jca.24985

39. Khozin S, Carson KR, Zhi J, Tucker M, Lee SE, Light DE, et al. Real-World Outcomes of Patients With Metastatic Non-Small Cell Lung Cancer Treated With Programmed Cell Death Protein 1 Inhibitors in the Year Following U.s. Regulatory Approval. Oncologist (2019) 24:648-56. doi: 10.1634/ theoncologist.2018-0307

40. Barlesi F, Dixmier A, Debieuvre D, Raspaud C, Auliac JB, Benoit N, et al. Effectiveness and Safety of Nivolumab in the Treatment of Lung Cancer Patients in France: Preliminary Results From the Real-World EVIDENS Study. OncoImmunology (2020) 9:1744898. doi: 10.1080/2162402X. 2020.1744898

41. Chen M, Li Q, Xu Y, Zhao J, Zhang L, Wei L, et al. Immunotherapy as SecondLine Treatment and Beyond for Non-Small Cell Lung Cancer in a Single Center of China: Outcomes, Toxicities, and Clinical Predictive Factors From a Real-World Retrospective Analysis. Thorac Cancer (2020) 11:1955-62. doi: 10.1111/1759-7714.13488

42. Morita R, Okishio K, Shimizu J, Saito H, Sakai H, Kim YH, et al. Real-World Effectiveness and Safety of Nivolumab in Patients With Non-Small Cell Lung Cancer: A Multicenter Retrospective Observational Study in Japan. Lung Cancer (2020) 140:8-18. doi: 10.1016/j.lungcan.2019.11.014

43. Weis TM, Hough S, Reddy HG, Daignault-Newton S, Kalemkerian GP. RealWorld Comparison of Immune Checkpoint Inhibitors in Non-Small Cell Lung Cancer Following Platinum-Based Chemotherapy. J Oncol Pharm Pract (2020) 26:564-71. doi: 10.1177/1078155219855127 
44. Loeb LA, Ernster VL, Warner KE, Abbotts J, Laszlo J. Smoking and Lung Cancer: An Overview. Cancer Res (1984) 44:5940-58.

45. Jung KJ, Jeon C, Jee SH. The Effect of Smoking on Lung Cancer: Ethnic Differences and the Smoking Paradox. Epidemiol Health (2016) 38:e2016060. doi: 10.4178/epih.e2016060

46. The Lancet Respiratory M. Lung Cancer Immunotherapy Biomarkers: Refine Not Reject. Lancet Respir Med (2018) 6:403. doi: 10.1016/s2213-2600(18) 30180-2

47. Sharpnack MF, Cho JH, Johnson TS, Otterson GA, Shields PG, Huang K, et al. Clinical and Molecular Correlates of Tumor Mutation Burden in Non-Small Cell Lung Cancer. Lung Cancer (2020) 146:36-41. doi: 10.1016/ j.lungcan.2020.05.021

48. Chae YK, Davis AA, Raparia K, Agte S, Pan A, Mohindra N, et al. Association of Tumor Mutational Burden With DNA Repair Mutations and Response to Anti-PD-1/PD-L1 Therapy in Non-Small-Cell Lung Cancer. Clin Lung Cancer (2019) 20:88-96.e6. doi: 10.1016/j.cllc.2018.09.008

49. Alexandrov LB, Nik-Zainal S, Wedge DC, Aparicio SA, Behjati S, Biankin AV, et al. Signatures of Mutational Processes in Human Cancer. Nature (2013) 500:415-21. doi: 10.1038/nature12477

50. Walser T, Cui X, Yanagawa J, Lee JM, Heinrich E, Lee G, et al. Smoking and Lung Cancer: The Role of Inflammation. Proc Am Thorac Soc (2008) 5:811-5. doi: 10.1513/pats.200809-100TH

51. Smolle E, Pichler M. Non-Smoking-Associated Lung Cancer: A Distinct Entity in Terms of Tumor Biology, Patient Characteristics and Impact of Hereditary Cancer Predisposition. Cancers (Basel) (2019) 11:204. doi: $10.3390 /$ cancers 11020204

52. Li X, Li J, Wu P, Zhou L, Lu B, Ying K, et al. Smoker and Non-Smoker Lung Adenocarcinoma Is Characterized by Distinct Tumor Immune Microenvironments. Oncoimmunology (2018) 7:e1494677. doi: 10.1080/ 2162402x.2018.1494677

53. Gonçalves RB, Coletta RD, Silvério KG, Benevides L, Casati MZ, da Silva JS, et al. Impact of Smoking on Inflammation: Overview of Molecular Mechanisms. Inflammation Res (2011) 60:409-24. doi: 10.1007/s00011-011-0308-7

54. Quail DF, Joyce JA. Microenvironmental Regulation of Tumor Progression and Metastasis. Nat Med (2013) 19:1423-37. doi: 10.1038/nm.3394

55. Yang DC, Chen CH. Cigarette Smoking-Mediated Macrophage Reprogramming: Mechanistic Insights and Therapeutic Implications. J Nat Sci (2018) 4:e539.
56. Rakaee M, Busund LR, Jamaly S, Paulsen EE, Richardsen E, Andersen S, et al. Prognostic Value of Macrophage Phenotypes in Resectable Non-Small Cell Lung Cancer Assessed by Multiplex Immunohistochemistry. Neoplasia (2019) 21:282-93. doi: 10.1016/j.neo.2019.01.005

57. Li B, Severson E, Pignon JC, Zhao H, Li T, Novak J, et al. Comprehensive Analyses of Tumor Immunity: Implications for Cancer Immunotherapy. Genome Biol (2016) 17:174. doi: 10.1186/s13059-016-1028-7

58. Ferrer I, Zugazagoitia J, Herbertz S, John W, Paz-Ares L, Schmid-Bindert G. KRAS-Mutant Non-Small Cell Lung Cancer: From Biology to Therapy. Lung Cancer (2018) 124:53-64. doi: 10.1016/j.lungcan.2018.07.013

59. Skoulidis F, Heymach JV. Co-Occurring Genomic Alterations in Non-SmallCell Lung Cancer Biology and Therapy. Nat Rev Cancer (2019) 19:495-509. doi: 10.1038/s41568-019-0179-8

60. Adderley H, Blackhall FH, Lindsay CR. KRAS-Mutant Non-Small Cell Lung Cancer: Converging Small Molecules and Immune Checkpoint Inhibition. EBioMedicine (2019) 41:711-6. doi: 10.1016/j.ebiom. 2019.02.049

61. Inamura K, Yokouchi Y, Kobayashi M, Sakakibara R, Ninomiya H, Subat S, et al. Tumor B7-H3 (CD276) Expression and Smoking History in Relation to Lung Adenocarcinoma Prognosis. Lung Cancer (2017) 103:44-51. doi: 10.1016/j.lungcan.2016.11.013

Conflict of Interest: The authors declare that the research was conducted in the absence of any commercial or financial relationships that could be construed as a potential conflict of interest.

Publisher's Note: All claims expressed in this article are solely those of the authors and do not necessarily represent those of their affiliated organizations, or those of the publisher, the editors and the reviewers. Any product that may be evaluated in this article, or claim that may be made by its manufacturer, is not guaranteed or endorsed by the publisher.

Copyright $\odot 2021$ Zhao, Jiang, Wang, He, Su and Yu. This is an open-access article distributed under the terms of the Creative Commons Attribution License (CC BY). The use, distribution or reproduction in other forums is permitted, provided the original author(s) and the copyright owner(s) are credited and that the original publication in this journal is cited, in accordance with accepted academic practice. No use, distribution or reproduction is permitted which does not comply with these terms. 